\title{
A Comparative Study on the Levels of Alkaline Phosphatase and Trace Elements in Gingival Crevicular Fluid and Serum of Periodontitis and Gingivitis Patients with Healthy Population
}

\author{
B.Meenakshi ${ }^{1}$, Chitraa R. Chandran ${ }^{1}$, T.R.Aravindhan ${ }^{1}$, Niranjali Devaraj ${ }^{2}$, \\ Rama K.V ${ }^{3}$. Valarmathi $S^{4}$. \\ ${ }^{I}$ Tagore Dental College and Hospital, Melakottaiyur, Chennai \\ ${ }^{2}$ University of Madras, Guindy, Chennai \\ ${ }^{3} I I T$, Madras, Guindy, Chennai \\ ${ }^{4}$ The TamilNadu Dr MGR Medical University, Guindy, Chennai
}

\section{Introduction}

Chronic periodontitis is a multifactorial disease that results in inflammation and destruction of the supporting structures of the tooth, mainly the periodontal ligament, cementum and alveolar bone (1). Traditional periodontal diagnostic procedures include probing depths, bleeding on probing, clinical attachment levels, plaque index and radiographs assessing alveolar bone level. All these clinical and radiographic parameters are difficult in identifying and measuring the progression of the disease (2). Research evidence suggest that altered activities of various enzymes, both at cellular and sub-cellular levels, can influence the disease process. Thus, measurements of enzyme activities can furnish valuable diagnostic and prognostic information $(3,4)$.

Elements such as selenium, copper and zinc are essential components of enzymes where they attract or substract molecules and faclilitate their conversion to specific end products. Copper plays an essential role in our metabolism largely by allowing many enzymes to function normally. Chromium is required for the biosynthesis of glucose tolerance factor (5-7). Zinc is required for the catalytic activity of a large number of enzymes. The enzyme most essential for zinc are alkaline phosphatase, alcoholdehydrogenase, carboanhydrase, glutamate and lactate dehydrogenase and RNA polymerases $(8,9)$. Zinc also plays a vital role in immune function, wound healing, synthesis of proteins, DNA and cell division(10,11). Zinc is necessary for proper sense of taste and smell(12). It plays the role of an antioxidant in the recovery of healing process after an injury and protection against aging (13).

Alkaline phosphatase (ALP) is an enzyme that plays a major role in bone homeostasis. It is present in liver, kidney, bone, intestine and placenta. It is also found in many cells of periodontium including neutrophils, osteoblasts and fibroblasts. ALP is released from polymorphonuclear neutrophils during inflammation, osteoblasts during bone formation and periodontal ligament fibroblasts during periodontal regeneration. Literature surveys have revealed the correlation of alkaline phosphatase in Gingival Crevicular Fluid with the severity of periodontitis(14).

A study by Malhotra et al (15) revealed that ALP can be considered as a periodontal disease marker as it can differentiate between healthy and inflamed sites. Another study had shown ALP level in GCF as a biomarker for the pathology and also an indicator of prognosis of periodontitis (16). Sanikop et al (17) stated that there is a relationship between ALP level and periodontal disease. This indicates that GCF ALP can be used as a potential biochemical marker for the detection and progression of periodontal diseases.

The objective of this study is to (i) compare the gingival crevicular fluid and serum alkaline phosphatase levels in periodontitis and gingivitis patients with healthy individuals and (ii) to compare the trace elements such as chromium, copper, selenium and zinc levels with alkaline phosphatase levels in gingival crevicular fluid and blood of periodontitis and gingivitis patients with healthy individuals.

\section{Materials And Methods}

This cross-sectional study was conducted from March 2013 to May 2014. Thirty six study subjects were recruited from the patients reporting to the Outpatient Department of Periodontics, Tagore Dental College, Chennai. The subjects were allowed to participate in the study if they have at least 20 teeth, excluding third molars. Both the sexes were allowed to participate in this study. Patients with a history of any systemic disease; subjects with history of intake of anti-inflammatory, antibiotics, antioxidants or multi-vitamin supplements in the previous six months, smokers, pregnant and lactating women were excluded.

The study was performed according to the Declaration of Helsinki, as revised in 2000, and was approved by the Institutional Ethical committee (IEC), Tagore Dental College. Informed consent was obtained from the participants who volunteered to participate in the study after explaining to them the study protocol in 
their regional language.

\section{Clinical examination}

All the selected individuals were subjected to a clinical examination done by a single examiner (18). Each individual underwent a full-mouth periodontal examination including the calculation of probing depth (PD), clinical attachment level (CAL) with a University of North Carolina (UNC)-15 periodontal probe (HuFriedy, Inc. Chicago, Illinois (IL), USA). The oral hygiene status and gingival health were assessed by taking Oral hygiene index(19) -simplified, gingivitis index and plaque index (Turesky Gillmore Glickman modification of Quigley-Hein plaque index).(20-22). Radiographic bone loss was recorded from intra oral periapical radiographs by long cone technique to differentiate chronic periodontitis group from the healthy individuals. The diagnosis of chronic periodontitis was made as per the criteria of Swati et al (23). The participants were then categorized into two groups; group I (healthy) consisted of 12 subjects with clinically healthy periodontium, with PD $<3 \mathrm{~mm}$ and no evidence of attachment loss and, group II (chronic periodontitis) consisted of 12 subjects who had signs of clinical inflammation, presence of PD $>4 \mathrm{~mm}$ in $30 \%$ of sites, clinical attachment loss $>1 \mathrm{~mm}$ in $30 \%$ of sites and radiographic evidence of bone loss.

\section{Selection of GCF collection site}

Samples were collected from only one site in each subject. GCF was pooled from one or more than two sites without any inflammatory signs in healthy participants to get an adequate volume. In periodontitis patients, sites were identified using a University of North Carolina (UNC)-15 periodontal probe (Hu-Friedy, Inc. Chicago, Illinois (IL), USA) and the site showing highest clinical signs of inflammation and highest CAL along with radiographic confirmation of bone loss was selected for sampling. Supragingival plaque was removed with sterile cuvettes without touching the marginal gingiva. The area was isolated with cotton to avoid the contamination of saliva. The GCF was collected by placing the micro capillary pipettes at the gingival sulcus and gently touching the marginal gingiva. A standardized $5 \mu 1$ volume of GCF was collected from each site using 1-5 $\mu \mathrm{l}$ calibrated volumetric microcapillary pipettes (Sigma -Aldrich, St.Louis , MO, USA). The sites from which there were no possibilities of GCF sample collection within the same time in the healthy individuals were excluded from the study. The micropipettes contaminated with blood and saliva were also excluded. The GCF collected was immediately transferred to a Eppenndorf tubes and stored at $-20{ }^{\circ} \mathrm{C}$ till further analysis.

\section{Collection of Serum}

Venous blood samples $(2 \mathrm{ml})$ were collected from the antecubital fossa by venipuncture using 20 gauge needle with $2 \mathrm{ml}$ syringes without EDTA and allowed to clot at room temperature. The samples were then centrifuged after one hour and the serum obtained was transferred to plastic vials and stored at $-20^{\circ} \mathrm{C}$ until further analyses of alkaline phosphatase and trace elements Copper, Zinc Selenium and Chromium were done.

\section{Biochemical Analysis}

The activity of ALP in GCF and serum was performed by the method of King and Armstrong (24), using UV visible spectrophotometer.

The estimation of trace elements such as Copper, Zinc, Selenium and Chromium in GCF and serum was done using Perkin Elmer optima 5300 Dual Viewing (DV) Inductively Coupled Plasma Optical Emission Spectrometer (ICPOES) $(25,26)$ at Sophisticated Analytical Instrument Facility (SAIF), Indian Institute of Technology, Chennai.

\section{Statistical analysis}

The activity of alkaline phosphatase and the levels of trace elements Copper, Zinc, Selenium and Chromium in GCF and serum of periodontitis, gingivitis and healthy individuals were tabulated and statistically analysed. Data are presented as mean \pm standard deviation. Mann - Whitney U test and Wilcoxon W tests were used.

\section{Results And Discussion}

Table 1 shows that the activity of ALP in both GCF and serum were found to be higher in gingivitis patients when compared to healthy individuals and periodontitis patients. The mean paired differences between chromium, copper, zinc and selenium and alkaline phosphatase levels in GCF of healthy, gingivitis and periodontitis patients are shown in table 2. The mean chromium, zinc and alkaline phosphatase levels were found to be higher in periodontitis patientswhen compared to healthy and gingivitis individuals. Copper and selenium levels were found to be negligible in gingivitis patients when compared to healthy individuals and periodontitis patients. 
A Comparative Study on the Levels of Alkaline Phosphatase and Trace Elements in ....

Table -1 Mean ALP levels in GCF and serum of Healthy, Gingivitis and Periodontitis subjects

\begin{tabular}{|l|l|l|l|}
\hline Samples & Healthy & Gingivitis & Periodontitis \\
\hline GCF(IU/L) & 0.021 & 0.050 & 0.003 \\
\hline SERUM (IU/L) & 0.006 & 0.012 & 0.004 \\
\hline
\end{tabular}

Trace elements such as chromium, copper, selenium and zinc play an important role in the functioning of many enzymes like superoxide dismutase, catalases, alkaline phosphatase etc (27). The levels of trace elements such as chromium, copper, selenium and zinc and ALP in GCF and serum of healthy individuals , gingivitis and periodontitis patients were estimated.

Table-2 Mean Paired differences between ALP and Trace Elements levels in GCF

\begin{tabular}{|l|l|l|l|}
\hline Parameter & Healthy ( p value) & Gingivitis(pvalue) & Periodontitis(pvalue) \\
\hline Chromium $(\mu \mathrm{g} / \mathrm{ml})$ & $0.307(0.08)$ & $0.053(0.62)$ & $0.337(0.05)$ \\
\hline Copper $(\mu \mathrm{g} / \mathrm{ml})$ & $0.034(0.10)$ & $0.007(0.79)$ & $0.098(0.00)$ \\
\hline Selenium $(\mu \mathrm{g} / \mathrm{ml})$ & $0.015(0.25)$ & $0.009(0.62)$ & $0.027(0.01)$ \\
\hline Zinc $(\mu \mathrm{g} / \mathrm{ml})$ & $0.051(0.04)$ & $0.045(0.25)$ & $0.558(0.10)$ \\
\hline
\end{tabular}

The mean GCF and serum alkaline phosphatase levels were found to be higher in gingivitis patients when compared to healthy individuals and periodontitis patients. This finding was similar to the study done by Malhotra et al (15) who reported statistically non significant increase in GCF ALP activity in gingivitis patients when compared to the healthy population.

A statistically significant increment $(p>0.00)$ in the level of serum copper and ALP was observed in periodontitis patients when compared to healthy individuals and gingivitis patients.

The mean level of chromium, zinc and alkaline phosphatase were found to be higher in periodontitis patients when compared to healthy individuals and gingivitis patients. Evidences for chromium deficiency have been observed especially in individuals with some degree of glucose intolerance. Chromium is necessary for biosynthesis of glucose tolerance factor $(28,29)$. The increased mean paired difference of alkaline phosphatase and chromium levels observed in the present study suggests that there is a posiibility that the raised levels can coexist with a negative imbalance. No comparison could be carried out as there were no similar studies done so far.

The levels of Copper, Selenium and ALP in GCF were found to be negligible in gingivitis patients when compared to healthy and periodontitis. Malhotra (15) had shown that GCF ALP activity was significantly increased in chronic periodontitis patients as compared to that of gingivitis patients. Research on Selenium and ALP activity in GCF is found to be scarce. GCF Zinc and ALP levels were found to be increased in periodontitis patients when compared to that of gingivitis and healthy individuals. This finding was similar to the report of Sanikop et al.,(17) who found an increased GCF ALP activity in gingivitis and periodontitis patients. Zinc has been established as an essential micro-nutrient in human health and nutrition. It is required for the functional activity of several enzymes including alkaline phosphatase (27). Not many studies are available on the association of GCF zinc and alkaline phosphatase levels .

Table-3 Mean Paired differences between ALP and Trace Elements levels in Serum

\begin{tabular}{|l|l|l|l|}
\hline Parameter & Healthy( p value) & Gingivitis(pvalue) & Periodontitis(pvalue) \\
\hline Chromium $(\mu \mathrm{g} / \mathrm{ml})$ & $0.113(0.25)$ & $0.010(0.07)$ & $0.173(0.08)$ \\
\hline Copper $(\mu \mathrm{g} / \mathrm{ml})$ & $0.730(0.00)$ & $0.782(0.00)$ & $0.829(0.00)$ \\
\hline Selenium $(\mu \mathrm{g} / \mathrm{ml})$ & $0.476(0.00)$ & $0.330(0.00)$ & $0.366(0.00)$ \\
\hline Zinc $(\mu \mathrm{g} / \mathrm{ml})$ & $0.347(0.00)$ & $0.405(0.00)$ & $0.472(0.00)$ \\
\hline
\end{tabular}

Chromium and ALP levels were found to be increased in periodontitis patients. Copper and ALP levels were found to be highest in all the three groups when compared to other trace elements. This is similar to the findings reported by Thomas B ( 30 ) who showed an increase in serum copper levels in periodontitis patients when compared with healthy individuals. Selenium and ALP levels were increased in healthy individuals when compared to that of gingivitis and periodontitis patients.

Our study is the first study to assess the levels of chromium with ICPOES method in the gingival crevicular fluid in healthy individuals and periodontitis subjects and to show an increased correlation with ALP. More research is therefore needed with an increased sample size to prove the correlation of ALP with trace elements in future. 


\section{References}

[1]. Offenbacher S. Periodontal diseases: Pathogenesis. Ann Periodontol 1996; 11:821-78

[2]. Fine DH. Incorporating new technologies in periodontal diagnosis into training programs and patient care : a critical assessment and a plan for the future. J Periodontol 1992; 63: 383-93.

[3]. Fine DH and Mandel ID. Indicators of periodontal disease activity. : An evaluation . J Clin Periodontol 1986;13: 533-46.

[4]. Todorovic T, Dozic I, Vicente-Barrero M, Ljuskovic B, Pejovic J, Marjanovic M , et al. Salivary enzymes and periodontal disease. Med Oral Patol Oral Cir Buccal 2006; 11: E 115-9. 5.Nielsen FH. New essential trace elements for the life sciences. Biol Trace Elem Res 1990;26-27:599-611.

[5]. Harris ED. Copper homeostasis. The role of cellular transporters. Nutr Rev2001;59:281-5.

[6]. Cefalu WT,Hu FB . Role of chromium in human health and in diabetes. Diabetes Care 2004; 27:2741-51.

[7]. H H. Sandstead. Understanding zinc: recent observations and interpretations.i Journal of Laboratory and Clinical Medicine 1994; vol 124, no3 ;322-27.

[8]. TJ McCarthy J, J Zeelie, DJ Krause. The antimicrobial action of zinc ion/antioxidant combinations. Journal of Clinical Pharmacy and Therapeutics 1992; vol 17, no $1 ; .51-54$

[9]. N W Solomons. Mild human zinc deficiency produces an imbalance between cell-mediated and humoral immunity. Nutrition Reviews 1998; vol 56, no 1; 27-28.

[10]. A S Prasad. Zinc: an overview. Nutrition 1995; vol 11, no1;93-99.

[11]. CA Heyneman. Zinc deficiency and taste disorders. Annals of Pharmacotherapy 1996; vol 30,no2;186-87.

[12]. N Fabris, E Mocchegiani. Zinc , human diseases and aging. Aging Clinical and Experimental Research 1995; vol 7, no 2;77-93

[13]. Cesco Rde T,Ito IY, de Albuquerque RF Jr. Levels of Aspartate Aminotransferase (AST) in saliva of patients with different periodontal conditions. J Clin Periodontol 2003; 30: 752-5. 15.Ranjan Malhotra , Vishakha Grover, Anoop Kapoor, Rupika Kapur.Alkaline phosphatase as a periodontal disease marker. Indian J Dent Res 2010; 21(4):531-536.

[14]. Jimly James Kunjappu,, Vinod Babu Mathew ,Shashikanth Hegde, Rajesh Kashyap and Rjesh Hosadurga. Assessment of the alkaline phosphatase level in gingival crevicular fluid, as a biomarker to evaluate the effect of scaling and root planning on chronic periodontitis : A in vivo study. J Oral Maxillofac Pathol 2012;Jan-Apr 16(1):54-57.

[15]. Sheetal Sanikop, Suvarna Patil, Pallavi Agrawal. Gingival crevicular fluid alkaline phosphatase as a potential diagnostic marker of periodontal diesae. Journal of Indian Society of Periodontology 2012;vol 16:4;513-518.

[16]. AravindhanThiruputkuzhiRanganathan, WaleedKhalid, Ponnandai Krishnamurthy Saraswathy, Chitraa Ramachandran,Lakshmiganthan Mahalingam. Periodontal findings in patients with Hunsens disease. Asian Pacific Journal of Tropical Biomedicine 2014; 4:suppl 2; S654-656.

[17]. Greene JC, Vermillion JR. The simplified oral hygiene index. J Am Dent Associ 1964 Jan; 68: 7-13

[18]. Silness $\mathbf{J}$ and Loe H. Periodontal diesease in pregnancy .II. Correlation between oral hygiene and periodontal condition .Acta Odontol Scand 1964; 22:112-135.

[19]. Loe H and Silness J. Periodontal disease in Pregnancy. Acta Odontologica Scandinavica (December) 1963; Vol 21:533-551.

[20]. Bissada NF, ScahafferEM, Haus E. Circadian periodicity of human crevicular fluid flow. J Periodontol 1967; 38: 36-4023.

[21]. Swati Pradeep Patel, Nishanth S Rao , AR Pradeep. Effect of nonsurgical periodontal therapy on crevicular fluid and serum glutathione peroxidase levels. Disease Markers 2012;32:1-7.

[22]. Earl J King and A Riley Armstrong. A convenient method for determining serum and bile phosphatase activity. The Canadian Medical Association Journal1934; 376-81.

[23]. Thompson \& Walsh.Handbook of Inductively Coupled Spectrometry. Thompson Press 1989; (India) Limited NewDelhi.

[24]. Charles B.Boss\& Kenneth J Fredeen. Concepts, Instrumentation andTechniques in Inductively Coupled Plasma Optical Emission Spectrometry(ICPOES) .2004; Third edition ; Perkin Elmer, Inc.

[25]. Nizel AE, Papas AS. Nutrition in Clinical Dentistry. $3^{\text {rd }}$ ed. Philadelphia Saunders: 1989.

[26]. Nicolas Wiernsperger, Jean Robert Rapin. Trace elements in gluco metabolic disorders:an update. Diabetology and Metabolic syndrome $2010 ; 2 ; 70$.

[27]. Pechova,A., Pavlata,L. Chromium is an essential nutrient :a review. Veterinarni Medicina.2007; 52 (1):1-18

[28]. Thomas B., Gautam A., Prasad BR., Kumari S. Evaluation of Micronutrient (zinc, copper and iron) levels in periodontitis patients with and Without diabetes mellitus type 2: a biochemical study. Indian J Dent Res 2013; 24(4):468-73. 